\title{
Surveillance Robot Using Multi Sensor Network
}

\author{
Pooventhan $\mathbf{K}^{1}$, Achuthaperumal $\mathbf{R}^{2}$, Kowshik $\mathbf{S}^{3}$, Manoj Balajee $\mathbf{C} \mathbf{R}^{4}$ \\ Assistant Professor, Electronics and Instrumentation, Sri Ramakrishna Engineering College, Coimbatore, India ${ }^{1}$ \\ UG Scholar, Electronics and Instrumentation, Sri Ramakrishna Engineering College, Coimbatore, India ${ }^{2,3,4}$
}

\begin{abstract}
The field of robotics is largely unexplored by the underdeveloped and the developing countries. Robotics have been a staple of advanced manufacturing for over half a century. Over the past decade, we have seen increasing levels of investment in autonomous vehicles used for surveillance and security. In domestic side the robots are employed only for detecting the human motion in the area and in capturing the motion and storing it in the database but in the defence side the robots are used for capturing the live motion and sending the live motion to the control room and capturing the person in the field. The proposed system focuses on designing a robot that can used for both defence and domestic systems. In this project embedded platform is used for making the robot, the robot which are we designing captures the live motion and the position of the live human using the camera and sends the live video and the position to the control room via wireless communication technique.
\end{abstract}

Keywords: Surveillance Robot, Security, Multi Sensor Network, Arduino.

\section{INTRODUCTION}

Surveillance is the monitoring of the behaviour, activities, or other changing information, usually of people for the purpose of influencing, managing, directing, or protecting them. Hence a robot which continuously monitor the place and provides security is developed. The first surveillance robot which is to be used for security purpose was "Mobile Detection. Assessment and Response System (MDARS)" Everett, H. \& Gage, D.W., 1999. From then there has been tremendous improvement in the research and development in surveillance robots. Now surveillance robots are used in all military and security applications. In this project a robot is designed in such way that it provides high level surveillance as required using automation. The main objective of the project is to provide an efficient surveillance wherever high level security is needed. The proposed system is an embedded based robotic module. With the proposed system, humans can feel extreme comfort and can experience automation to the maximum.

\section{SURVEILLANCE ROBOT AND MULTI SENSOR NETWORKSURVEILLANCEROBOT}

The first surveillance robot which is to be used for security purpose was "Mobile Detection Assessment and Response System (MDARS)" Everett, H. \& Gage, D.W., 1999. From then there has been tremendous improvement in the research and development in surveillance robots. Now surveillance robots are used in all military and security applications. Basically surveillance robot is nothing but the ordinary robot with the navigation mechanism along with some cameras, thermal sensors, and the communication devices likeGSM, GPS modules. These robots can be navigated using servo motors to get the accurate navigation.

The overall setup needed for the project is shown in the Fig. 1. In the above diagram shown PIR refers to the Passive Infrared Sensor, GSM and GPS refers to Global System for Mobile communication and Global Positioning System respectively.

\section{A. Overall block diagram and description}
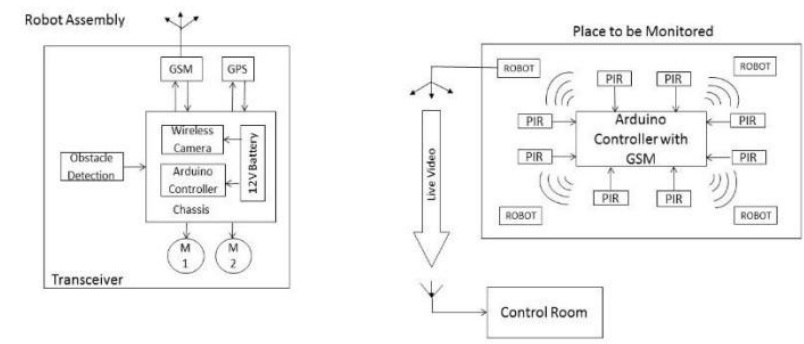

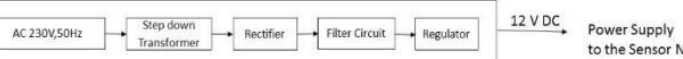

Fig. 1. Overall Block diagram of the setup B. Description

First the sensor network is created wherever the high level surveillance is needed. PIR sensors are used for the motion detection purpose.

The sensor network is made to communicate with the robots with the help of GSM communication.

Whenever a sensor detects a motion then it will send a text message to the robots which are nearer to the sensor using GSM communication.

Now the robots which have received the text message will rover the area around the sensor network.

While robot is on rover, live video will be transmitted to the control room over internet. If the robot detect live human then it will send the location of that human will also be shared with the control room.

C. Parts of the robot navigation mechanism Each and every robot need to be navigated to some other place. To do this we can use various mechanisms for this robot we used servo motors for the Navigation of the robot.

\section{Controller unit}

Controller unit is the heart of the robot. For this project we used Arduino UNO microcontroller kit to control the robot according to the various sensor outputs. 


\section{E. Wireless IP camera}

Wireless IP camera is used for the live video transmission over the internet. It uses the Internet Protocol to transmit the images and the videos captured/recorded. This camera is mounted on the surveillance robot.

\section{F. Power supply}

It is the major need for the surveillance robot to function in a proper manner. The surveillance robot need $12 \mathrm{~V} \mathrm{DC}$ input for proper functioning. So $12 \mathrm{~V}$ battery is mounted on the robot for mobile applications. It is a rechargeable battery.

\section{G. Communication system}

Communication system is used to make the robot communicate with other units like Control room, Sensor Network etc., Basically the communication system consist of a transmitter(GSM, Wireless Camera) and receiver(GPS,GSM). Here the GSM is used as the Transceiver.

\section{H. Robotic sensors}

A sensor is an electronic device that transfers a physical phenomenon into an electrical signal. Sensors are the sensory system of a robot, like the five sensors that humans have: Touch, Sound, Sight, Smell, and Taste. Sensors also measure environmental data like touch, distance, light, sound, strain, rotation, magnetism, smell, temperature, inclination, pressure, or altitude. For this project PIR and IR sensors are used for live motion detection and for obstacle detection respectively.

\section{Multi sensor network}

Multi Sensor Network is nothing but the Network created by the sensors which will be connected to a controller. The controller reads the data of all the sensors. Each sensor has a unique Identity or the address so that the controller will identify which data is belong to which sensor. Here Arduino UNO is used as the Controller of the Sensor Network. A program is written on the controller so that it collects all the sensor's data and sends a message to the robot that a particular sensor detected some motion. PIR (Passive Infra-Red) sensor network is created to detect the live motion.

\section{III.RESULTS}

The surveillance robot is assembled successfully and the test run was successful. As mentioned earlier surveillance robot consists of Navigation mechanism and wireless camera (Android mobile phone) on it.

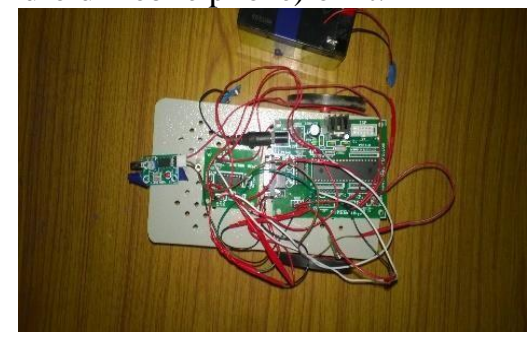

Fig.2. Top view of the robot

The Fig. 2. Shows the top view of the designed robot assembly. In this robot the AVR development board is used as the navigation controller. A $12 \mathrm{~V}$ battery is used as the power source.

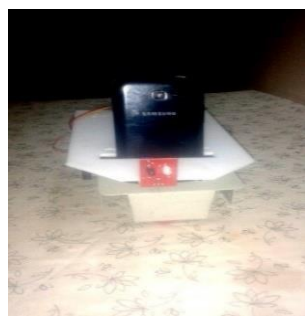

Fig. 3. Front view of the robot

Front view of the robot is shown in the Fig.3. The camera (in this case the mobile phone) is placed in front of the robot in order to capture the video. IR sensor is fixed at edge of the chassis for the purpose of obstacle detection.

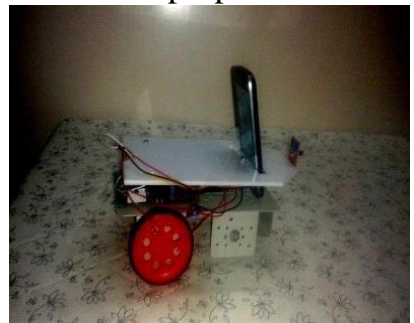

Fig. 4. Side view of the robot

The side view of the robot assembly is shown in Fig.4. DC motors are used for navigation purpose of the robot. The robot is programmed in Embedded $\mathrm{C}$ language such that if it detects any obstacle it will take left direction from the current direction.

\section{IV.CONCLUSION}

The surveillance robot was designed with AVR microcontroller using embedded platform. It monitors and secure a place from the adversaries which can be done by surveillance robot all the times with great accuracy and high precision. An IP camera is used which continuously monitors the place and sends the information to the control station. The Servo motor used provides the movement of robot with greater speed control compared to the conventional method. The future scope of the project has many openings that could be continued for various future applications in monitoring and controlling etc., This robot can also be used in time of environmental disasters where the robot detects whether a human is present alive in that area. Domestic applications like Home security can also be implemented using this methodology.

\section{REFERENCES}

[1] Birk A. \& Kenn H. (2001) 'An industrial application of behaviour oriented robotics', International Conference on Robotics and Automation, Volume 1, pp. 749-754.

[2] Capezio F., Sgorbissa A. \& Zaccaria R. (2005) 'GPS Based Localization for a Surveillance UGV in Outdoor Areas',Proceedings of the FifthInternational Workshop on Robot Motion and Control (RoMoCo'05), pp. 157- 162, ISBN: 837143-266-6.

[3] Dehuai Z., Gang X., Jinming Z. \& Li L. (2007) 'Development of a mobile platform for security robot', Proceedings of IEEE International Conference on Automation and Logistics, pp. 12621267, ISBN: 978-1-4244-1531-1.

[4] Donato Di Paola, Annalisa Milella, Grazia Cicirelli and Arcangelo Distante (2010) 'An Autonomous Mobile Robotic System for Surveillance of Indoor Environments', International Journal of Advanced Robotic Systems, ISSN 1729-8806 Volume 7, No. 1, pp. 019-026. 
[5] Krishnaswamy Kannan, Gowtham.S, Adithya.R (2012) 'Intelligent Personal Assistant and Surveillance Robot using ZigBee Communication'. International Journal of Engineering Science and Technology (IJEST), Volume 4, Issue 4, pp. 278-290.

[6] Kulyukin V., Gharpure C., Nicholson J. \& Pavithran S. (2004) 'RFID in Robot-Assisted Indoor Navigation for the Visually Impaired', IEEE/RSJ International Conference on Intelligent Robots and Systems, pp. 1979-1984.

[7] Milella A., Cicirelli G. \& Distante A. (2008) 'RFID Assisted Mobile Robot System for Mapping and Surveillance of Indoor Environments', An International Journal, Vol. 35, No. 2, pp. 143-152.

[8] Pratheepa.S, Dr.Purushothaman Srinivasan (2011)'Surveillance Robot For Tracking Multiple Moving Targets', International Journal of Scientific \& Engineering Research, Volume 2, Issue 3, pp. 789-800.

[9] Sivasoundari.A, Kalaimani.S, Balamurugan.M (2013) 'Wireless Surveillance Robot with Motion Detection and Live Video Transmission', International Journal of Emerging Science and Engineering (IJESE) Volume 1, Issue-6, pp. 266-278.

[10] Vig L. \& Adams J.A. (2007) 'Coalition formation: From software agents to robots', Journal of Intelligent and Robotic Systems, Volume 50, No. 1, pp. 85-118, ISSN: 0921-0296. 